\title{
The Relationship Between TNF-Like Protein 1A and Coronary Artery Aneurysms in Children With Kawasaki Disease
}

\section{jing zhang}

Chongqing Medical University Affiliated Children's Hospital https://orcid.org/0000-0002-9848-7763 Haobo Weng

Chongqing Medical University Affiliated Children's Hospital

\section{Qiongfei Pei}

Chongqing Medical University Affiliated Children's Hospital

\section{Penghui Yang}

Chongqing Medical University Affiliated Children's Hospital

\section{Wentao Fan}

Chongqing Medical University Affiliated Children's Hospital

\section{Ruixi Liu}

Department of Cardiovascular Medcine, Children's Hospital of Chongqing Medical University, China. Qijian Yi ( $\nabla$ qjyi2003@Hotmail.com )

Department of Cardiovascular Medicine, Children's Hospital of Chongqing Medical University, China.

\section{Research Article}

Keywords: Tumor necrosis factor (TNF)-like protein 1A, Kawasaki disease, Coronary artery aneurysm, Clinical parameters

Posted Date: March 11th, 2021

DOl: https://doi.org/10.21203/rs.3.rs-274389/v1

License: (c) (i) This work is licensed under a Creative Commons Attribution 4.0 International License. Read Full License

Version of Record: A version of this preprint was published at Clinical and Experimental Medicine on July 7th, 2021. See the published version at https://doi.org/10.1007/s10238-021-00729-5. 


\section{Abstract}

Background: Kawasaki disease (KD) is an acute, systemic vasculitis of unknown etiology that occurs predominantly in infants and children, and the most crucial complication of KD is coronary artery aneurysm (CAA). Tumor necrosis factor (TNF)-like protein 1A (TL1A) is a member of the TNF superfamily, which possesses the ability of maintaining vascular homeostasis and regulating immune response. This study aims to examine the serum TL1A levels in KD patients, and to investigate the relationship between TL1A and CAAs in children with KD.

Methods: Blood samples were recruited from 119 KD patients, 35 febrile controls (FCs) and 37 healthy controls (HCs). The KD group was further divided into KD with CAAs (KD-CAAs) and KD non-CAAs (KDNCAAs) groups. Serum TL1A levels were measured using enzyme-linked immunosorbent assays, and clinical parameters were collected in KD patients.

Results: Serum TL1A levels in the acute phase of KD patients were significantly higher than that in the FC and HC groups. In particular, serum TL1A were substantially increased in the KD-CAA group than that in the KD-NCAA group. Furthermore, TL1A levels were positively correlated with the duration of fever, time point of IVIG and WBC levels, but negatively correlated with levels of RBC, $\mathrm{Hb}$ and Albumin in the KD group.

Conclusions: TL1A might be involved in the KD-associated vasculitis, and might be a factor in the development process of CAAs.

\section{Introduction}

Kawasaki disease (KD) has been defined as an acute, systemic vasculitis that mainly occurs in infants and children aged $<5$ years ${ }^{[1]}$. It predominantly affects the systemic medium-sized blood vessels. The most critical complication of KD is coronary artery lesions (CALs). Indeed, KD has replaced rheumatic fever as the leading cause of pediatric acquired cardiac disease in developed countries. Although a single dose of $2 \mathrm{~g} / \mathrm{kg}$ intravenous immunoglobulin (IVIG) combining with aspirin, is now considered as the standard treatment strategy, there are still about $15 \%$ children with KD developing CALs or coronary artery aneurysms (CAA), which may lead to coronary artery stenosis or thrombosis, ischemic heart disease or even myocardial infarction and sudden death ${ }^{[2,3]}$. The etiology and pathogenesis of KD are still unclear. In recent years, several studies have suggested that endothelial dysfunction and excessive inflammatory responses playing a key role in the pathogenesis of $\mathrm{KD}^{[4]}$.

Tumor necrosis factor (TNF)-like protein 1A (TL1A), also known as vascular endothelial growth inhibitor, is a member of the TNF superfamily. It has a high degree of sequence homology with TNF-a ${ }^{[5]}$. TL1A is primarily produced by endothelial cells under stimulation of TNF- $a$ and IL-1, and is also expressed in the lymphocytes, macrophages, dendritic cells, chondrocytes and synovial cells ${ }^{[6]}$. TL1A could stimulate T cell activation, proliferation, and production of large amounts of essential cytokines via death receptor 3 
(DR3), which is expressed mainly on lymphocytes ${ }^{[5]}$.TL1A plays an important role in apoptosis, modulation of immune responses, vascular homeostasis, glucose and lipid metabolism ${ }^{[5,7,8]}$. McLaren et $\mathrm{al}^{\left[{ }^{[9]}\right.}$ have demonstrated that TL1A promotes the formation of foam cell in vitro by regulating the levels of low-density lipoprotein and cholesterol that are involved in atherosclerosis. Recent evidences also demonstrated a relationship between serum TL1A and the occurrence of coronary artery disease and acute coronary syndrome ${ }^{[10,11]}$ and serum TL1A might be a specific biomarker for monitoring of coronary artery disease. In addition, TL1A can promote the expression of MiR-29b by activating the JNK-GATA3 signaling pathway ${ }^{[12]}$, and MiR-29b further mediated the down-regulation of extracellular matrix proteins, which played a key role in development of aortic dilatation and aortic aneurysm ${ }^{[13]}$. But there is still no report on the relationship between TL1A and CAAs in children with KD.

\section{Methods}

\section{Patients and their General Characteristics}

This study enrolled 119 children with acute phase of KD from the Children's Hospital of Chongqing Medical University, Chongqing, China. All patients ( 80 males and 39 females, $2.228 \pm 1.591$ years old) met the guidelines proposed by the Japanese Kawasaki Disease Research Committee ${ }^{[14]}$. The subjects with a previous history of KD, metabolic diseases or any other associated immunological disease were excluded from this study. 35 subjects ( 23 males and 12 females, $2.388 \pm 2.285$ years old) with acute febrile disease were recruited as a febrile control (FC) group and 37 physical examinations children (24 males and 13 females, $3.657 \pm 0.551$ years old) as a healthy control (HC) group. FC subjects included patients with bronchopneumonia, infectious diarrhea, urinary tract infection, sepsis, hand-foot-mouth disease (HFMD), infectious mononucleosis, scarlet fever, suppurative meningitis, herpangina. This study was approved by the Ethics Committee of Children's Hospital, Chongqing Medical University and informed consent was obtained from the parents or appropriate guardians.

Echocardiography was measured on the KD patients 1 day before administration of intravenous immunoglobulin (IVIG) or anticoagulants. Subjects with a z-value $\geq 2.5$ were included in the KD with CAA group (KD-CAAs, $n=59$ ), while those with $z$-value $<2.5$ were included in the KD non-CAA group (KD-NCAAs, $\mathrm{n}=60)^{[15]}$.

\section{Blood sample collection}

All blood samples were collected 1 day before treatment with IVIG or anticoagulants in the KD patient group. The blood samples were immediately centrifuged at 3,000 rpm for $10 \mathrm{~min}$ to get the serum and were stored at $-80^{\circ} \mathrm{C}$ at once. The same procedure was followed to obtain serum samples from the FCs and HCs.

\section{Measurement of serum TL1A concentrations and clinical parameters}


Serum TL1A levels were measured using an enzyme-linked immunosorbent assay (ELISA) according to the manufacturer's instructions (Cloud-Clone Corp, China).

Important clinical data was recorded and clinical parameters of the blood samples were also measured, including the duration of fever, the time point of IVIG, white blood cell count (WBC), percentage of neutrophils (N\%), percentage of leukomonocytes (L\%), red blood cell count (RBC), hematocrit(HCT), hemoglobin $(\mathrm{Hb})$, platelet counts (PLT), mean platelet volume (MPV), platelet distribution width (PDW), Creactive protein (CRP), erythrocyte sedimentation rate (ESR), procalcitonin(PCT), albumin (ALB), aspartate aminotransferase (AST), alanine aminotransferase (ALT), and creatine kinase-MB (CK-MB).

\section{Statistical Analysis}

All statistical analyses were evaluated using the SPSS25.0 software for Windows (SPSS, Chicago, IL). A two-sided $P$ value $<0.05$ was considered statistically significant. $\chi^{2}$-test were used to compare frequencies between groups. Differences between groups were assessed using the unpaired 2-tailed $t-$ test. Spearman rank correlation was used to analyze the association between serum TL1A levels and various clinical parameters. All values are quoted as mean \pm SD or number and percent $(n, \%)$. Presence of KD development was expressed by area under curve (AUC) using receiver operating characteristic (ROC) analysis.

\section{Results}

\section{Serum TL1A levels in all Study Subjects}

Serum TL1A levels were significantly higher in the KD group $(191.2 \pm 100 \mathrm{pg} / \mathrm{ml}, \mathrm{n}=119)$ than that in the $\mathrm{FC}$ (82.79 $\pm 49.73 \mathrm{pg} / \mathrm{ml}, \mathrm{n}=35)$ or $\mathrm{HC}(70.93 \pm 31.6 \mathrm{pg} / \mathrm{ml}, \mathrm{n}=38)$ groups. (Fig.1)

\section{TL1A levels and clinical parameters in the KD-CAA and KD-NCAA groups}

There were no significant differences in duration of fever, the time point of IVIG, WBC, RBC, PLT, Albumin, MPV, PDW, CRP, ESR, AST, ALT, or CK-MB between the KD-CAA and KD-NCAA groups ( $P>0.05$ ). But, compared to the KD-NCAA group, the levels of $\mathrm{Hb}$ and N\% were decreased and the levels of TL1A and $L \%$ were increased in the KD-CAA group $(P<0.05)$. (Table 1$)$

\section{Correlations between TL1A levels and clinical parameters in the KD Group}

In the KD group, N\%, L\%, MPV, PDW, ALT, AST, CK-MB, CRP, ESR were not significantly correlated with serum TL1A levels. However, serum TL1A levels were positively correlated with the duration of fever, time point of IVIG, WBC and PLT levels $(\mathrm{p}<0.05)$, and were negatively correlated with RBC, $\mathrm{Hb}$ and Albumin $(P<$ 0.05 ) in the KD group. (Table 2)

\section{Correlations between TL1A levels and clinical parameters in the KD-CAA and KD-NCAA groups}


Serum TL1A levels were positively correlated with the duration of fever, WBC, PLT And CRP levels in the KD-NCAA group. $(P<0.05)$, and were not significantly correlated with the duration of fever, time point of IVIG, WBC, Hb, N\%, L\%, MPV, PDW, ALT, AST, CK-MB, CRP, ESR, were positively correlated with PLT levels $(\mathrm{P}=0.000)$, and were negatively correlated with RBC and Albumin. $(P<0.05)$ in the KD-CAA group. (Table 3)

\section{ROC curves of TL1A used to predict Kawasaki disease (KD)}

The AUC value of KD was 0.8678 (95\% confidence interval $0.8175-0.9180, P<0.0001)$, compared to both FC and HC groups. The cut-off value of KD was 123.4 , with sensitivity of $88.89 \%$ and specificity of 75.63\%. (Fig.2)

\section{Discussion}

It has been reported that TL1A can regulate metabolism ${ }^{[8,16]}$ in physiological status and play an important role in the modulation of vascular homeostasis and autoimmune diseases ${ }^{[5,7]}$. The pathogenesis of KD is well known as inflammation and imbalance of immunology resulting in vascular lesion. The purpose of this study was to ascertain the relationship between TL1A and CAA in children with KD. The results showed that serum TL1A levels were significantly higher in KD patients than those in the FC and HC groups. Serum TL1A levels were much higher in the KD-CAA group than that in the KDNCAA group. Furthermore, TL1A levels were positively correlated with the duration of fever, time point of IVIG, WBC and PLT levels, but negatively correlated with RBC, $\mathrm{Hb}$ and Albumin in the KD group.

TL1A, an important molecule related to inflammation, is characterized by a metalloprotease cleavage site and a C-terminal TNF homology domain ${ }^{[17]}$. It has been shown that the levels of TL1A were significantly increased in patients with acute coronary syndrome, and associated with both endothelial dysfunction and coronary atherosclerosis ${ }^{[11]}$. Recent studies have shown that TL1A promotes foam cell formation in vitro by regulating the levels of low-density lipoprotein and cholesterol ${ }^{[9]}$, and it switches vascular smooth muscle cell phenotypes to participate in the development of atherosclerosis in the mouse models for apolipoprotein E-deficient atherosclerosis ${ }^{[18]}$. Here we found that the levels of TL1A in the acute phase of $\mathrm{KD}$ patients was significantly higher than those in $\mathrm{FC}$ and $\mathrm{HC}$ group, indicating that TL1A might be act as a proinflammatory factor in the vasculitis of $K D$. There were lots of researches demonstrated that as a $T$ cell co-stimulator, TL1A plays an important role in the immune response. Studies have shown that increased levels of TNF- $a$, IL-1 $\beta$, IL-2R IL-6and IFN- $\gamma$ are involved in the inflammation associated with the development of CALs, and TL1A can promote secretion of these cytokines ${ }^{[5,19-22]}$. According to above results we speculated that TL1A might be a key factor for the excessive inflammatory responses of KD. In addition, TL1A induces apoptosis of endothelial cells in an autocrine manner and activates a series of signaling pathways, such as SAPK/JNK, p38 MAPK and NF-KB signaling pathway ${ }^{[23]}$. It induces dysfunction and injury of coronary endothelial cells in the acute stage of KD, which lead to the dilatation of coronary arteries. And inflammation-mediated lesions gradually develop in the whole arterial layer, especially smooth muscle layer, eventually destruction of the vascular wall's supporting system and 
formation of CAA ${ }^{[24]}$. Studies have found that TL1A promotes the expression of MiR-29b via JNKGATA3 $^{[12]}$. MiR-29b further destroys the integrity of the vascular wall by targeting the extracellular matrix protein and inducing smooth muscle cell apoptosis accelerates the formation of aneurysms ${ }^{[13]}$. Taken together, all those results indicated that TL1A might be involved in the inflammatory process and CAA in patients with $\mathrm{KD}$.

In this study, we found that the levels of PLT in KD-CAA group was higher than that in the KD-NCAA group, although, this difference was not statistically significant. Serum TL1A levels were positively correlated with PLT in KD group, especially in the KD-CAA group. Previous studies have reported that TL1A was able to enhance PDGF-BB ${ }^{[20]}$, which produced mainly by platelet. It seemly hinted that TL1A may be involved in the formation of thrombus in children with KD-CAA.

Anemia and hypoproteinemia are common clinical features of KD patients ${ }^{[25,26]}$. In this study, the levels of albumin and hemoglobin in the KD-CAA group were lower than those in the KD-NCAA group, and TL1A levels in KD patients were negatively correlated with the levels of albumin and hemoglobin. Studies have shown that TL1A can increase reactive oxygen to accelerate inflammatory response via TL1A/TNFR2 pathway ${ }^{[27]}$. Straface et $a^{[28]}$ have demonstrated that systemic oxidative stress and premature and senescence of RBC may play a key role in anemia and hypoproteinemia in children with KD, so TL1A may promote the changes of $\mathrm{RBC}$ and $\mathrm{Hb}$ in $\mathrm{KD}$ patients. On the other hand, albumin loss might result from the increased vascular permeability accompanied with the endothelium damage ${ }^{[26]}$. TL1A can induce endothelial cell apoptosis and excessive inflammation leading to destroy of vascular barrier ${ }^{[5]}$. Our study showed a significantly higher TL1A serum levels in the KD-CAA group than that in the KD-NCAA group, while the serum albumin levels were negatively associated with TL1A. These results suggests that hypoalbuminemia could reflect the extent of the vascular damage, and high serum concentration of TL1A might be one of causes of hypoalbuminemia in KD.

When compared to both FC and HC Groups, the AUC value was 0.8678 ( $95 \%$ confidence interval 0.8175$0.9180, p<0.0001)$ in KD group. The cut-off value of serum TL1A levels was $123.4 \mathrm{pg} / \mathrm{mL}$ and the sensitivity and specificity of predicting were $88.89 \%$ and $75.63 \%$, respectively. This provides further evidence that TL1A also may play an important role in the development of KD.

However, there are limitations in our study, such as it was a single-center study and the number of participants were relatively small, the results may be some bias. Additionally, due to lack of data on serum TL1A levels at different time points of patients with KD, we were unable to reveal the trend of TL1A changes or analyze the recovery of KD-associated vasculitis.

\section{Conclusion}

In summary, our study was the first to demonstrate that the serum TL1A was higher in KD patients than that in FCs and HCs groups. And serum TL1A levels were substantially increased in the KD-CAA group when compared to the KD-NCAA group. Serum TL1A levels were correlated with RBC and Albumin levels 
KD group. These results indicated that TL1A might be involved in the KD-associated vasculitis, and TL1A might be involved in the development of CAAs in children with KD. Further studies about the mechanism of TL1A in the development KD and CAAs should be performed.

\section{Declarations}

\section{Acknowledgments:}

We would like to appreciate the recruited children for their kindness to support the development of medicine.

Funding: This study was funded by Excellent Youth Foundation of Chongqing Scientific Committee: 81500273.

Conflicts of interest: The authors have no conflicts of interest to declare that are relevant to the content of this article.

\section{Authors' contributions:}

Jing Zhang has substantial contributions to conception and design of this study, acquisition of data, analysis of data and drafting the article.

Haobo Weng contributes to design of this study, interpretation of data and revise the article.

Qiongfei Pei contributes to acquisition and interpretation of data.

Penghui Yang contributes to acquisition and interpretation of data.

Wentao Fan contributes to acquisition of data.

Ruixi Liu contributes to design of this study, acquisition and interpretation of data.

Qijian Yi* has substantial contributions to revising it critically for important intellectual content and final approval of the version to be published.

Consent to participate: All authors agreed to participate in the study.

Consent for publication: Manuscript is approved by all authors for publication.

\section{References}

1. Newburger JW, Takahashi M, Burns JC. Kawasaki Disease. J Am Coll Cardiol. 2016, 67(14): 17381749.

2. Kato H, Ichinose E, Yoshioka F, et al. Fate of coronary aneurysms in Kawasaki disease: serial coronary angiography and long-term follow-up study. Am J Cardiol. 1982, 49(7): 1758-1766. 
3. McCrindle BW, Rowley AH, Newburger JW, et al. Diagnosis, Treatment, and Long-Term Management of Kawasaki Disease: A Scientific Statement for Health Professionals From the American Heart Association. Circulation. 2017;135(17):e927-99.

4. Koibuchi H, Kotani K, Minami T, et al. Endothelial dysfunction by flow-mediated dilation assessed ultrasonically in patients with Kawasaki Disease. Minerva Pediatr. 2016, 68(2): 143-147.

5. Zhang Z, Li LY. TNFSF15 Modulates Neovascularization and Inflammation. Cancer Microenviron. 2012, 5(3): 237-247.

6. Migone TS, Zhang J, Luo X, et al. TL1A is a TNF-like ligand for DR3 and TR6/DcR3 and functions as a T cell costimulator. Immunity. 2002, 16(3): 479-492.

7. Aiba Y, Nakamura M. The role of TL1A and DR3 in autoimmune and inflammatory diseases. Mediators Inflamm. 2013, 2013: 258164.

8. Maixner N, Pecht T, Haim Y, et al. A TRAIL-TL1A Paracrine Network Involving Adipocytes, Macrophages, and Lymphocytes Induces Adipose Tissue Dysfunction Downstream of E2F1 in Human Obesity. Diabetes. 2020,69(11): 2310-2323.

9. McLaren JE, Calder CJ, McSharry BP, et al. The TNF-like protein 1A-death receptor 3 pathway promotes macrophage foam cell formation in vitro. J Immunol. 2010;184(10):5827-34.

10. Chen X, Guo Y, Lai L, et al. Intracoronary and peripheral blood levels of TNF-like Cytokine 1A (TL1A) in patients with acute coronary syndrome. Medicine (Baltimore). 2020, 99(22): e20305.

11. Stamatelopoulos K, Georgiou S, Kanakakis I, et al. Circulating levels of TNF-like cytokine 1A correlate with reflected waves and atherosclerosis extent and may predict cardiac death in patients with stable coronary artery disease. Cytokine. 2015, 72(1): 102-104.

12. Zhang K, Cai HX, Gao S, et al. TNFSF15 suppresses VEGF production in endothelial cells by stimulating miR-29b expression via activation of JNK-GATA3 signals. Oncotarget. 2016, 7(43): 69436-69449.

13. Boon RA, Seeger T, Heydt S, et al. MicroRNA-29 in aortic dilation: implications for aneurysm formation. Circ Res. 2011, 109(10): 1115-1119.

14. Fukazawa R, Kobayashi J, Ayusawa M, et al. JCS/JSCS 2020 Guideline on Diagnosis and Management of Cardiovascular Sequelae in Kawasaki Disease. Circ J. 2020, 84(8): 1348-1407.

15. Manlhiot C, Millar K, Golding F, et al. Improved classification of coronary artery abnormalities based only on coronary artery z-scores after Kawasaki disease. Pediatr Cardiol. 2010, 31(2): 242-249.

16. Yu M, Lu G, Zhu X, et al. Downregulation of VEGF and upregulation of TL1A expression induce HUVEC apoptosis in response to high glucose stimuli. Mol Med Rep. 2016, 13(4): 3265-3272.

17. Richard AC, Ferdinand JR, Meylan F, et al. The TNF-family cytokine TL1A: from lymphocyte costimulator to disease co-conspirator. J Leukoc Biol. 2015, 98(3): 333-345.

18. Zhao D, Li J, Xue C, et al. TL1A inhibits atherosclerosis in apoE-deficient mice by regulating the phenotype of vascular smooth muscle cells. J Biol Chem. 2020,295(48): 16314-16327. 
19. Ma Z, Wang B, Wang M, et al. TL1A increased IL-6 production on fibroblast-like synoviocytes by preferentially activating TNF receptor 2 in rheumatoid arthritis. Cytokine. 2016, 83: 92-98.

20. Guo J, Luo Y, Yin F, et al. Overexpression of Tumor Necrosis Factor-Like Ligand 1 A in Myeloid Cells Aggravates Liver Fibrosis in Mice. J Immunol Res. 2019, 2019: 7657294.

21. Teraura $H$, Kotani $K$, Minami $T$, et al. The serum concentration of soluble interleukin-2 receptor in patients with Kawasaki disease. Ann Clin Biochem. 2017, 54(2): 209-213.

22. Feng $S$, Su Y, Luo L, et al. Serum levels of $C 1 q /$ tumor necrosis factor-related protein-1 in children with Kawasaki disease. Pediatr Res. 2018, 83(5): 999-1003.

23. Yue TL, Ni J, Romanic AM, et al. TL1, a novel tumor necrosis factor-like cytokine, induces apoptosis in endothelial cells. Involvement of activation of stress protein kinases (stress-activated protein kinase and p38 mitogen-activated protein kinase) and caspase-3-like protease. J Biol Chem. 1999, 274(3): 1479-1486.

24. He YE, Qiu HX, Wu RZ, et al. Oxidised Low-Density Lipoprotein and Its Receptor-Mediated Endothelial Dysfunction Are Associated with Coronary Artery Lesions in Kawasaki Disease. J Cardiovasc Transl Res. 2020, 13(2): 204-214.

25. Huang YH, Kuo HC. Anemia in Kawasaki Disease: Hepcidin as a Potential Biomarker. Int J Mol Sci. 2017, 18(4): 820.

26. Terai $\mathrm{M}$, Honda $\mathrm{T}$, Yasukawa $\mathrm{K}$, et al. Prognostic impact of vascular leakage in acute Kawasaki disease. Circulation. 2003, 108(3): 325-330.

27. Al-Azab M, Qaed E, Ouyang X, et al. TL1A/TNFR2-mediated mitochondrial dysfunction of fibroblastlike synoviocytes increases inflammatory response in patients with rheumatoid arthritis via reactive oxygen species generation. FEBS J. 2020, 287(14): 3088-3104.

28. Straface E, Gambardella L, Metere A, et al. Oxidative stress and defective platelet apoptosis in naïve patients with Kawasaki disease. Biochem Biophys Res Commun. 2010, 392(3): 426-430.

\section{Tables}

Table 1. TL1A levels and Clinical parameters in the KD-CAA and KD-NCAA groups 


\begin{tabular}{|llll|}
\hline & KD-CAAs $(\mathrm{n}=59)$ & KD-NCAAs $(\mathrm{n}=60)$ & P value \\
\hline Duration of fever (day) & $7.356 \pm 2.565$ & $6.483 \pm 1.321$ & 0.121 \\
\hline Time point of & $7.153 \pm 2.864$ & $6.661 \pm 2.814$ & 0.430 \\
IVIG (day) & & & \\
\hline WBC $\left(10^{3} / \mu \mathrm{l}\right)$ & $14.670 \pm 3.722$ & $14.470 \pm 4.951$ & 0.495 \\
\hline RBC $\left(10^{3} / \mu \mathrm{l}\right)$ & $4.058 \pm 0.414$ & $4.087 \pm 0.332$ & 0.529 \\
\hline PLT $\left(10^{3} / \mu \mathrm{l}\right)$ & $422.500 \pm 178.700$ & $376.600 \pm 119.800$ & 0.330 \\
\hline Albumin $(\mathrm{g} / \mathrm{L})$ & $35.750 \pm 4.643$ & $37.250 \pm 4.094$ & 0.076 \\
\hline Hb $(\mathrm{g} / \mathrm{L})$ & $104.900 \pm 12.310$ & $109.200 \pm 7.759$ & $0.043^{*}$ \\
\hline N\% & $0.607 \pm 0.138$ & $0.670 \pm 0.144$ & $0.019 *$ \\
\hline L\% & $0.316 \pm 0.119$ & $0.267 \pm 0.128$ & $0.028 *$ \\
\hline MPV (fl) & $9.851 \pm 0.756$ & $9.640 \pm 0.843$ & 0.074 \\
\hline PDW (fl) & $10.930 \pm 1.507$ & $10.440 \pm 1.585$ & $0.038^{*}$ \\
\hline ALT (U/l) & $54.800 \pm 59.820$ & $44.840 \pm 54.960$ & 0.163 \\
\hline AST (U/l) & $35.100 \pm 35.220$ & $38.630 \pm 38.540$ & 0.474 \\
\hline CK-MB (U/l) & $0.979 \pm 0.628$ & $1.200 \pm 0.974$ & 0.178 \\
\hline CRP (mg/dl) & $51.270 \pm 30.180$ & $49.860 \pm 32.180$ & 0.639 \\
\hline ESR (mm/h) & $68.820 \pm 27.970$ & $69.210 \pm 24.560$ & 0.692 \\
\hline TL1A (pg/ml) & $211.000 \pm 108.500$ & $171.700 \pm 87.540$ & $0.044^{*}$ \\
\hline
\end{tabular}

Note: KD, Kawasaki disease; CAA, coronary artery aneurysm; NCAA, non-CAA; WBC, white blood cell counts; RBC, red blood cell counts; PLT, platelet counts; Hb, hemoglobin; N\%, percentage of neutrophils; $L \%$, percentage of leukomonocytes; MPV, mean platelet volume; $P D W$, platelet distribution width; $A L T$, alanine aminotransferase; $A S T$, aspartate aminotransferase; $C K-M B$, creatine kinase-MB; CRP, C-reactive protein; ESR, erythrocyte sedimentation rate. ${ }^{*} P<0.05$

Table 2. Correlations between TL1A levels and clinical parameters in the KD Group 


\begin{tabular}{|lll|}
\hline & \multicolumn{3}{l}{ TL1A } \\
\cline { 2 - 3 } & $r$ & $p$ \\
\hline Duration of fever (day) & 0.229 & $0.012^{*}$ \\
\hline Time point of IVIG (day) & 0.211 & $0.022^{*}$ \\
\hline WBC $\left(10^{3} / \mu \mathrm{l}\right)$ & 0.273 & $0.003^{*}$ \\
\hline RBC $\left(10^{3} / \mu \mathrm{l}\right)$ & -0.256 & $0.005^{*}$ \\
\hline PLT $\left(10^{3} / \mu \mathrm{l}\right)$ & 0.430 & $0.000^{*}$ \\
\hline Albumin $(\mathrm{g} / \mathrm{L})$ & -0.314 & $0.001 *$ \\
\hline Hb $(\mathrm{g} / \mathrm{L})$ & -0.247 & $0.007 *$ \\
\hline N\% & 0.108 & 0.243 \\
\hline L\% & -0.107 & 0.248 \\
\hline MPV $(\mathrm{fl})$ & -0.013 & 0.890 \\
\hline PDW (fl) & 0.021 & 0.827 \\
\hline ALT (U/l) & -0.107 & 0.245 \\
\hline AST (U/l) & -0.153 & 0.097 \\
\hline CK-MB $(\mathrm{U} / \mathrm{l})$ & -0.109 & 0.245 \\
\hline CRP (mg/dl) & 0.178 & 0.056 \\
\hline ESR (mm/h) & 0.062 & 0.549 \\
\hline
\end{tabular}

Note: KD, Kawasaki disease; CAA, coronary artery aneurysm; NCAA, non-CAA; WBC, white blood cell counts; RBC, red blood cell counts; PLT, platelet counts; Hb, hemoglobin; $N \%$, percentage of neutrophils; $L \%$, percentage of leukomonocytes; MPV, mean platelet volume; PDW, platelet distribution width; $A L T$, alanine aminotransferase; $A S T$, aspartate aminotransferase; $C K-M B$, creatine kinase-MB; $C R P, C$-reactive protein; ESR, erythrocyte sedimentation rate. ${ }^{*} P<0.05$

Table 3. Correlations between TL1A levels and clinical parameters in the KD-CAA and KD-NCAA groups 


\begin{tabular}{|lllll|}
\hline & \multicolumn{2}{l}{ KD-CAAs $(\mathrm{n}=59)$} & \multicolumn{2}{l}{ KD-NCAAs $(\mathrm{n}=60)$} \\
\cline { 2 - 5 } & $\mathrm{r}$ & $\mathrm{p}$ & $\mathrm{r}$ & $\mathrm{p}$ \\
\hline Duration of fever (day) & 0.153 & 0.246 & 0.309 & $0.016^{*}$ \\
\hline Time point of IVIG (day) & 0.148 & 0.262 & 0.264 & $0.043^{*}$ \\
\hline WBC $\left(10^{3} / \mu \mathrm{l}\right)$ & 0.220 & 0.093 & 0.339 & $0.008^{*}$ \\
\hline RBC $\left(10^{3} / \mathrm{\mu l}\right)$ & -0.345 & $0.007 *$ & -0.113 & 0.392 \\
\hline PLT $\left(10^{3} / \mu \mathrm{l}\right)$ & 0.487 & $0.000 *$ & 0.282 & $0.029 *$ \\
\hline Albumin (g/L) & -0.352 & $0.006 *$ & -0.207 & 0.113 \\
\hline Hb (g/L) & -0.208 & 0.113 & -0.235 & 0.071 \\
\hline N\% & 0.207 & 0.116 & 0.102 & 0.437 \\
\hline L\% & -0.203 & 0.124 & -0.094 & 0.473 \\
\hline MPV (fl) & -0.146 & 0.277 & 0.070 & 0.604 \\
\hline PDW (fl) & -0.088 & 0.517 & 0.072 & 0.590 \\
\hline ALT (U/l) & -0.035 & 0.795 & -0.251 & 0.053 \\
\hline AST (U/l) & -0.088 & 0.509 & -0.205 & 0.117 \\
\hline CK-MB (U/l) & -0.089 & 0.512 & -0.090 & 0.500 \\
\hline CRP (mg/dl) & 0.109 & 0.425 & 0.257 & $0.049 *$ \\
\hline ESR (mm/h) & 0.167 & 0.251 & -0.086 & 0.566 \\
\hline
\end{tabular}

Note: KD, Kawasaki disease; CAA, coronary artery aneurysm; NCAA, non-CAA; WBC, white blood cell counts; RBC, red blood cell counts; PLT, platelet counts; Hb, hemoglobin; $N \%$, percentage of neutrophils; $L \%$, percentage of leukomonocytes; MPV, mean platelet volume; PDW, platelet distribution width; $A L T$, alanine aminotransferase; $A S T$, aspartate aminotransferase; $C K-M B$, creatine kinase-MB; $C R P, C$-reactive protein; ESR, erythrocyte sedimentation rate. ${ }^{*} P<0.05$

\section{Figures}




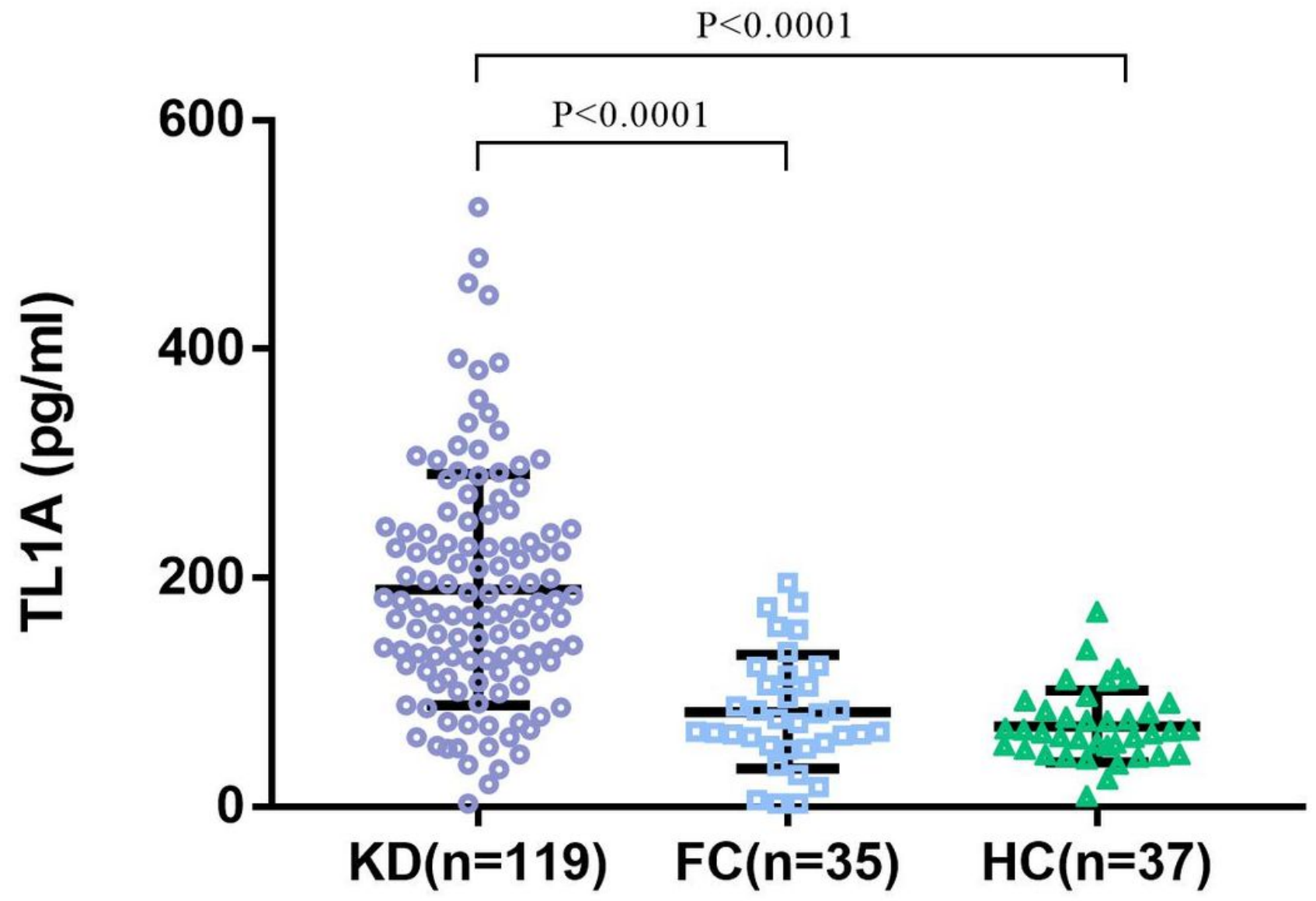

Figure 1

Serum TL1A levels in all subjects. KD Kawasaki disease, FC Febrile control, HC Healthy control. 


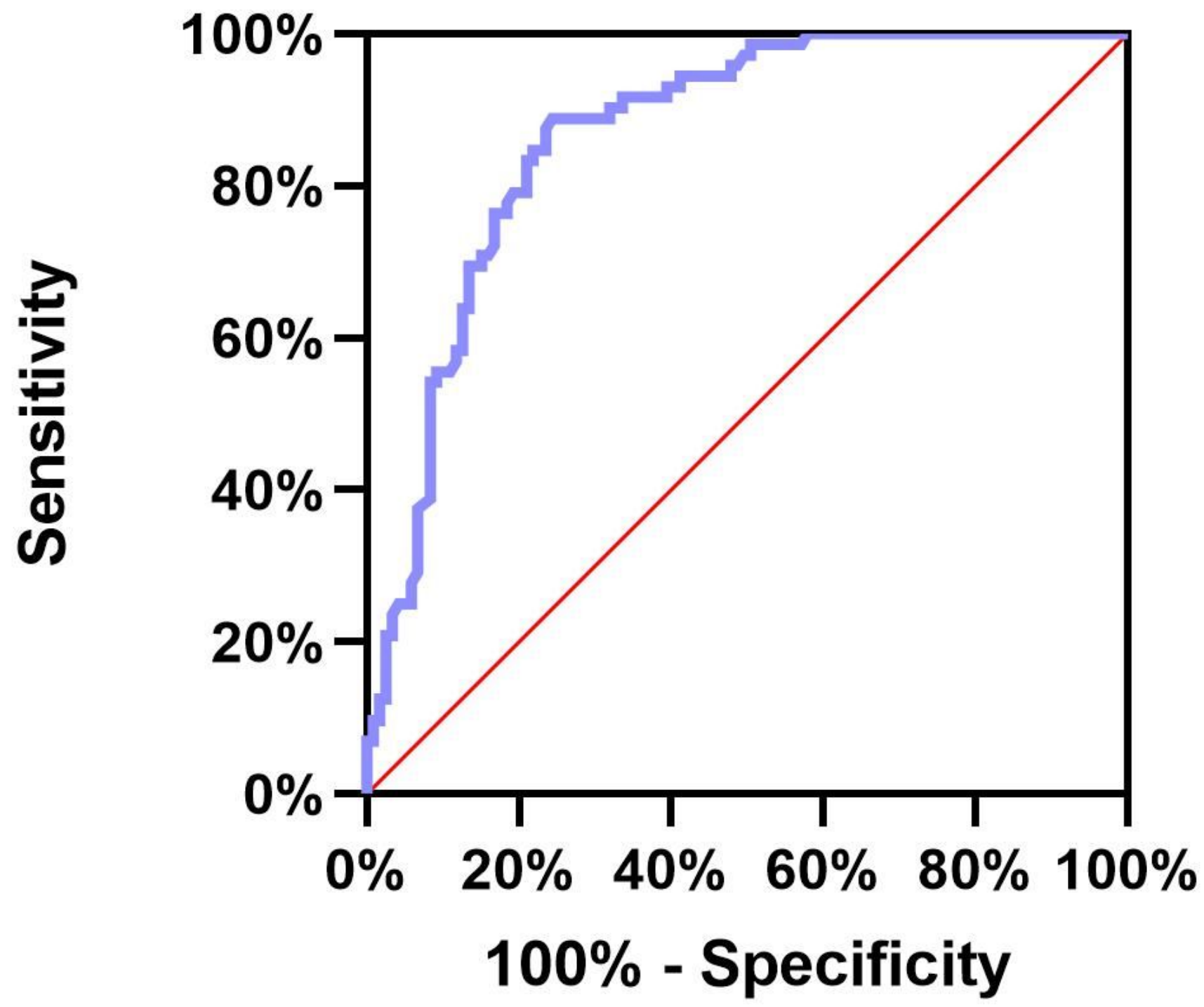

Figure 2

ROC curves of TL1A 